or, $\log \frac{I_{\mathrm{Na}}}{I_{\mathrm{Li}}}=(m R+n)(1-k)+\log \frac{q}{p}\left(\frac{d-b}{a-c}\right)$.

Since $a, b, c, d, m, n, p$, and $q$ are constants, this equation may be written as

$$
\log \frac{I_{\mathrm{Na}}}{I_{\mathrm{Li}}}=s R+t,
$$

where $s$ and $t$ are fresh constants.

It is evident from equations 4 and 5 that the slope of the calibration curve $\left(\log \left(I_{\mathrm{Na}} / I_{\mathrm{Li}}\right)\right.$ versus $\left.R\right)$ depends solely on properties of the colour wedge. The smallest value of $\bar{I}_{\mathrm{Na}} / I_{\mathrm{Li}}$ for which a null point can, theoretically, be obtained is found by putting $T_{\mathrm{Li}}=T_{\mathrm{Na}}=1$ in equation $\mathrm{I}$, and is equal to $\frac{q}{p}\left(\frac{d-b}{a-c}\right)$. This value depends on properties of the photo-cell and of the rotating filters, but does not depend on properties of the colour wedge. In practice, however, the null point must be straddled by taking readings on either side; further, the extremè tip of the wedge is not usable, so that the smallest measurable value of the light intensity ratio is somewhat higher than this calculation would suggest.

This system of photometry makes minimal demands on the properties of the components. Thus allowance has been made in the calculation for the fact that the colour wedge may absorb lithium light to some extent ; it is therefore unnecessary to search for a solution which absorbs sodium light but transmits lithium light without attenuation. Further, the rotating filters do not have to effect a perfect separation of the two wave-lengths ; indeed, the system will work so long as one of the rotating filters transmits a greater fraction of the sodium light and a smaller fraction of the lithium light than the other. The measurements are independent of fluctuations of amplifier gain, and of non-linearity in the amplifier ; they depend solely on properties of components of the optical system, which are unlikely to change from day to day. It is possible to work at low intensities of light since the amplifier does not have to deal with direct current, and it is practicable, therefore, to use several stages of amplification. In addition, it is probable that temperature and fatigue effects are minimized since the comparison is effected on a single photosensitive surface.

A separate publication by one of us (E. G. W.) will deal with the practical application of this method to flame photometry.

Nuffield Department of Clinical Medicine,

E. G. WALSH Radeliffe Infirmary, Oxford.

H. S. WOLFF

Medical Research Council Pneumoconiosis Unit, Cardiff. Nov. 24.

\section{Variations in the Length of the Day}

THE article by R. I. B. Cooper ${ }^{1}$ describes, inter alia, an investigation made by us to determine the effect of change in the ocean's moment of inertia, due to seasonal changes in sea-level, upon the periodic fluctuation in the length of the day.

In this investigation, from a study of the anomalies about mean sea-level, the increase of the ocean's moment of inertia for the spring period centred on March-April was computed to be $1.03 \times 10^{38} \mathrm{gm} . \mathrm{cm} .^{2}$. This value was used in conjunction with Munk's figure of $-0.4 \times 10^{36} \mathrm{gm} . \mathrm{cm} .^{2}$ for January, and assuming a sinusoidal distribution for the change of inertia with time, the maximum inertia change occurs at the end of April. The effect of this is about three months out of phase with the observed effect, and would therefore contribute very little.

In view of this, a further investigation has been carried out in which Munk's value for January was discarded, and a recomputation for January-February made on the same basis as for March-April. A value of $1.22 \times 10^{36} \mathrm{gm} . \mathrm{cm}^{2}$ was obtained, and combining this with the March-April figure results in an absolute maximum value of $1.64 \times 10^{36} \mathrm{gm} . \mathrm{cm} .^{2}$, occurring at the beginning of February. This result is precisely in phase with the total effect, and is therefore a direct contribution. Moreover, the accumulative effect in time is 0.01 sec. (approximately), which is more than 15 per cent of the observed effect.

A. I. LAWFORD

V. F. C. VELEY

Hydrographic Department, Admiralty. March 30.

${ }^{1}$ Cooper, R. I. B., Nature, 167, 298 (195i).

\section{Measurement of the Production of Organic Matter in the Sea by means of Carbon-14}

IT has often been suggested that by far the greatest production of organic matter on earth is due to the marine plankton algæ. Investigations are, however, extremely scarce. In some few areas the quantities of organic matter produced have been calculated by means of indirect methods, as, for example, from the decrease of nutrient salts or carbon dioxide during a definite period. The quantities calculated are, at best, minimum values for production, as account could be taken neither of the carbon dioxide absorbed from the atmosphere or produced by respiration of animals and bacteria, nor of the nutrient salts regenerated during the period and utilized again ${ }^{1}$.

A very few coastal waters have been investigated using a proper method for giving the production of matter directly. The only method employed hitherto has been that devised by Gran ${ }^{2}$ of suspending samples of sea water containing its natural plankton in the sea. The increase of the oxygen content is estimated after a certain period of time, mostly $24 \mathrm{hr}$. The determination of oxygen in the water is carried out with greatest accuracy when the production of matter is being measured in productive coastal waters. In oligotrophic waters and in waters with a great depth of the photic zone (production calculated per volume of water therefore small) Gran's method is unserviceable.

When preparing for the Danish oceanographical expedition with the Galathea, which was to cover all the oceans, it was therefore found necessary to devise a new method. The radioactive tracer carbon14 now available made this possible. Preparatory tests were made in Copenhagen during 1950. The method has now been used for a considerable time on board the Galathea. It has worked successfully both in very eutrophic and in very oligotrophic waters.

During the cruise of the Galathea in the eastern part of the Atlantic from Lisbon to Cape Town, the production of organic matter was measured by this tracer method at fifty-eight stations altogether. The productivity of the ocean in this area was found to vary between about $20 \mathrm{mgm}$. carbon (near Teneriffe) and about 2,000 $\mathrm{mgm}$. carbon (Benguela Current) 